12 Word count (including abstract): 4762/12000

13 Abstract: $132 / 150$

14
Running head: Introducing the Causal Graph Approach to Psychopathology

\section{Introducing the Causal Graph Approach to Psychopathology: An Illustration in} Patients with Obsessive-Compulsive Disorder

\author{
Jolanda J. Kossakowski, \\ Lucas J. B. van Oudheusden \\ Richard J. McNally \\ Bradley C. Riemann \\ Lourens J. Waldorp \\ Han L. J. van der Maas
}


Introducing the Causal Graph Approach to Psychopathology

Abstract

36

37 Clinicians aim to discern causal relations among features of mental disorders. Yet

38 observational data are insufficient to confirm these relations. In the present study, we

39 describe a causal graph approach to illustrate how one can discern causal relations among

40 features of obsessive-compulsive disorder (OCD) by applying the Invariant Causal

41 Prediction (ICP) algorithm and the Hidden Invariant Causal Prediction (HICP) algorithm,

42 supplemented by findings from a literature review. Applying these methods to symptom

43 severity data from four time points (before, during [twice], and after behavioral treatment) we

44 estimated a causal graph for OCD. The resulting causal graph reveals multiple cycles

45 between aspects of OCD that may play a role in the maintenance of the disorder. We

46 conclude that the proposed design has great potential and could benefit clinicians with some

47 adjustments and extensions.

48 
49 Introducing the Causal Graph Approach to Psychopathology: An Illustration in Patients with 50 Obsessive-Compulsive Disorder

51

52

53

54

55

56

57

58

59

60

61

62

63

64

65

66

67

68

69

70

71

72

73

74

75

76
The network perspective is an emerging view that postulates that mental disorders form a system of interactive symptoms. Instead of a latent variable - the "underlying disorder" - causing symptoms that presumably reflect its presence, the symptoms and their interactions constitute the disorder, rendering the latent variable entirely redundant (Borsboom, 2017; Borsboom \& Cramer, 2013). Figure 1 illustrates a causal graph: a network where the symptoms (here $X, Y$ and $Z$.) are depicted as nodes (the circles), and the causal relations between symptoms are represented by an arrow (directed edge).

Clinical researchers aim to identify causal relations among symptoms. Unfortunately, their aspirations are often stymied by mere correlational data that can suggest, but never confirm, causal relations. Indeed, experimental manipulations designed to underwrite causal conclusions are frequently infeasible in clinical settings. However, new methods have emerged that enable such inferences (Kossakowski, Waldorp, \& van der Maas, 2019). The purpose of our article is to introduce these methods by applying them to patients with obsessive-compulsive disorder (OCD).

In this study, we define a causal relation as a relation between two variables $X \rightarrow Y$ where, when we manipulate (or intervene on) variable $X$, we observe a change in variable $Y$ (conditioned on all other variables; Kossakowski et al., 2019). This definition also means that, if we do not observe any changes in $Y$, no intervention has taken place on $X$. When it comes to the treatment of patients, it is important to know whether symptom $X$ causes symptom $Y$, or perhaps vice versa. Figure 1 (left panel) illustrates a simple example where we have three variables, $X \rightarrow Y \rightarrow Z$. If the causal relations among these three variables are unknown, a therapist may first therapeutically target variable $Z$. However, as shown in this illustration, it may be more effective to target variables $X$ and $Y$, as both of these have an (in)direct effect on variable $Z$. 

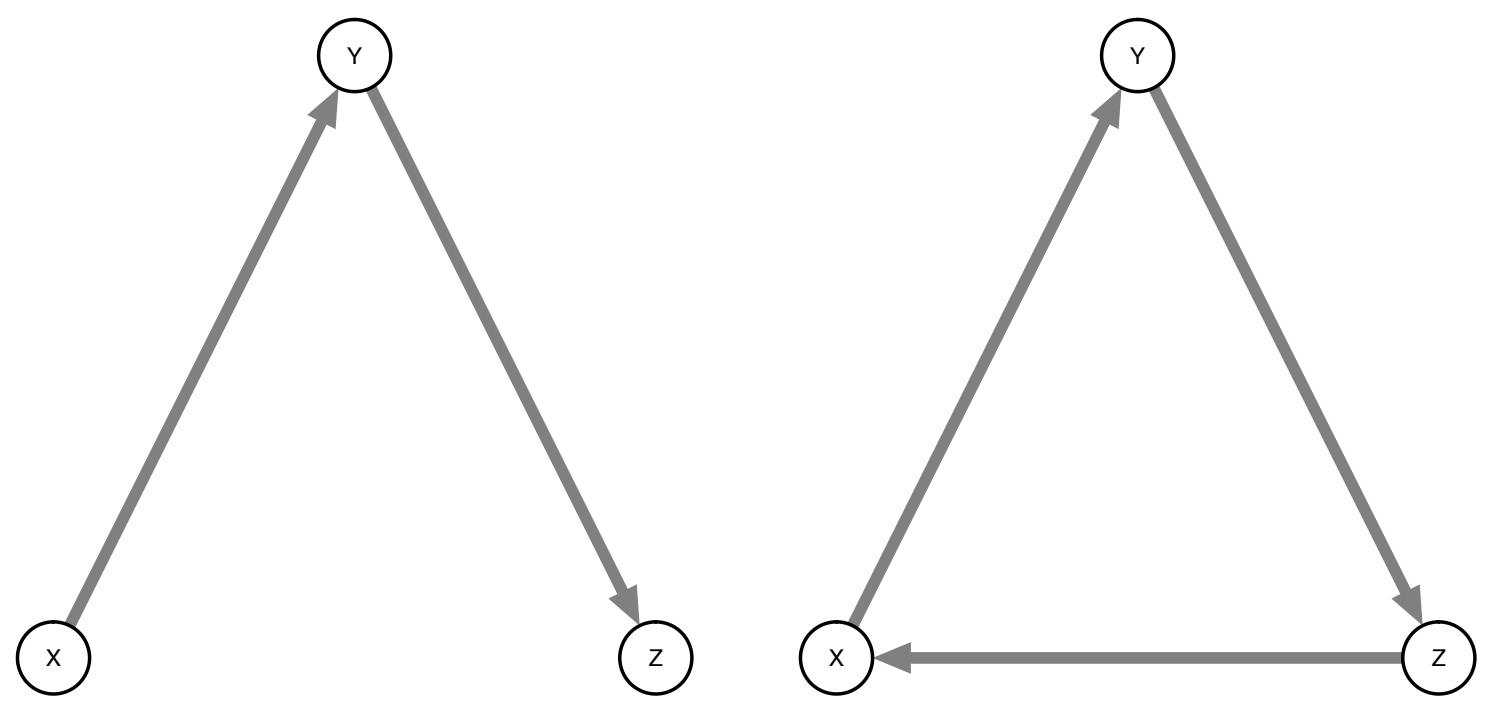

78 Figure 1. Illustration of causal relations among three variables $X, Y$, and $Z$. The left panel

Many studies use observational data to estimate causal relations between variables. Observational data are data on which no interventions have taken place. However, observational data alone do not allow one to estimate causal relations accurately; one needs to manipulate variables to estimate true causal relations (Holland, 1986). Thus, we need interventional data upon which some manipulation or intervention has taken place. Intervention here indicates that some manipulation is performed compared to the observational data. Examples include starting treatment or reducing one's medication. By using both observational and interventional data, we could potentially estimate true causal relations between symptoms (Meinshausen et al., 2016).

In this study, we describe a causal graph approach in which we combine observational and interventional data to estimate a causal graph. This approach is illustrated with an application to data of patients diagnosed with OCD who were measured at four time points: before, twice during, and after treatment. OCD is characterized by intrusive, persistent thoughts, urges or images (obsessions), and repetitive behaviors or mental acts (compulsions) performed to relieve the distress that accompanies the obsessions (American Psychiatric Association [APA], 2013). US national and global life-time prevalence rates for OCD range from between $1.6 \%$ to $2.3 \%$ (e.g., Fontenelle, Mendlowicz, \& Versiani, 2006; Kessler et al., 2005; Ruscio, Stein, Chiu, \& Kessler, 2010). Two network studies on OCD estimated 
100 Directed Acyclic Graphs (DAGs) involving features of OCD and symptoms of depression

101 (Jones, Mair, Riemann, Mugno, \& McNally, 2018; McNally, Mair, Mugno, \& Riemann,

102 2017). A DAG illustrates potential predictive causal relations among symptoms that together

103 depict a causal system (Pearl \& Mackenzie, 2018), but only if two stringent assumptions are

104 met. The causal graph must contain all important variables, and there cannot be any cycles

105 among the nodes. A cycle is shown in Figure 1 (right panel), where variable $\mathrm{Z}$ is the cause of

106 variable $\mathrm{X}$, thereby creating a cycle.

107 Ideally, to estimate causal relations, we would want to manipulate one symptom at a

108 time, and observe its effects on other symptoms. This approach would mimic an approach in

109 gene biology whereby researchers "knock-out" or silence a specific gene and observe the

110 subsequent effects (Pinna, Soranzo, \& de la Fuente, 2010; Serra, Villani, \& Semeria, 2004).

111 Using a "slim finger," geneticists can deactivate a gene while leaving all other genes

112 untouched. Unfortunately, clinical psychologists are saddled with "fat fingers" that render it

113 difficult to deactivate a single symptom without simultaneously affecting other symptoms.

114 For example, it would be nearly impossible to administer a benzodiazepine for insomnia

115 without also reducing anxiety at the same time. Ironically, targeting one symptom (e.g.,

116 insomnia) will not only affect other symptoms in serial fashion (e.g., less fatigue the

117 following day), but will also produce effects on additional symptoms in parallel fashion (e.g.,

118 anxiety), thereby complicating causal analysis. Indeed, algorithms designed for gene knock-

119 out experiments may not work as accurately when applied to psychological data

120 (Kossakowski et al., 2019).

121 In the causal graph approach, we applied the Invariant Causal Prediction (ICP)

122 algorithm (Meinshausen et al., 2016) and the Hidden Invariant Causal Prediction (HICP)

123 algorithm (Peters, Buhlmann, \& Meinshausen, 2016; Peters, Janzing, \& Schölkopf, 2017).

124 These algorithms represent a significant advance over DAGs as the daunting assumptions of

125 the latter are difficult to meet in clinical science. The ICP-algorithm can handle interventional

126 data where multiple symptoms are simultaneously manipulated. The HICP-algorithm

127 complements the ICP-algorithm because it can accommodate hidden variables - unmeasured

128 variables that may affect the measured variables nonetheless (Peters et al., 2016, 2017). Both

129 algorithms have shown great potential for psychological applications (Kossakowski et al.,

130 2019). Lastly, following Meinshausen et al.'s (2016) approach, we also searched the literature

131 for evidence of causal relations between features of OCD. We combined results from the

132 ICP-algorithm, the HICP-algorithm and the literature review into a single causal graph that

133 may give an overview of the causal structure of OCD. 


\section{Participants}

137 We used archival clinical data from patients with a primary OCD diagnosis. A total of

1383474 patients entered the study at one of the ten locations of the Rogers Behavioral Health

139 centers across the United States treated between June 2012 and June 2018. Patients received

140 treatment either in an intensive outpatient $(n=1320)$, partial hospitalization $(n=1247)$, or in

141 a residential unit $(n=906)$. The treatment setting for one patient was unknown. The primary

142 intervention was exposure and response prevention therapy. We included only those patients

143 who completed the relevant questionnaires prior to treatment, at the first and second progress

144 assessments, and the post-treatment assessment ( $t=4$ time points). We ended up with 903

145 patients that were included for all analyses. Patients gave their written informed consent to

146 use de-identified data for research purposes. Of this sample, 282 patients received treatment

147 in an intensive outpatient unit, 371 patients received treatment in a partial hospitalization

148 unit, and 250 patients received treatment in a residential unit. One patient switched from an

149 intensive outpatient unit to a partial hospitalization unit after the initial measurement. The

150 average number of days between admission and discharge was 56.39 ( $S D=20.29$ days). The

151 average time between measurements ranged from 15.81 to 23.25 days, with a standard

152 deviation ranging from 7.43 to 17.67 days. The sample consisted of 478 (52.93\%) females

153 and $424(46.95 \%)$ males, whose age ranged from 18 to $76(M=29.69, S D=11.42)$. The

154 gender of one participant was unknown. Based on the recommendations presented by Mataix-

155 Cols et al. (2016), who argue that a treatment response is shown by a reduction in Y-BOCS

156 score by at least 35\% compared to their score at baseline, 538 patients in our sample

157 experienced a clinically meaningful reduction in symptoms.

159 Yale-Brown Obsessive-Compulsive Scale Self-Report

160 Patients completed a test battery that contained, among other questionnaires, the Yale-

161 Brown Obsessive-Compulsive Scale Self-Report (Y-BOCS-SR; Steketee, Frost, \& Bogart,

162 1996), the self-report version of the Y-BOCS (Goodman, Price, Rasmussen, Mazure,

163 Fleischmann, et al., 1989). Patients completed the Y-BOCS-SR at four time points before,

164 during, and after treatment. The Y-BOCS-SR is a 10-item questionnaire that evaluates

165 features of OCD without taking the content of a patient's obsessions and compulsions into

166 account (e.g., harming versus contamination obsessions; checking versus washing

167 compulsions). The Y-BOCS-SR has a symmetric structure: the questionnaire evaluates five 
168 aspects of obsessions and of compulsions, respectively. Table 1 gives a more specific

169 overview of the individual items, including the means and standard deviations of each item

170 per measurement. All items were measured on a five-point Likert scale, ranging from 0 (no

171 symptoms) to 4 (extreme). The Y-BOCS-SR shows excellent psychometric properties (e.g.,

172 Goodman, Price, Rasmussen, Mazure, Delgado, et al., 1989; Goodman, Price, Rasmussen,

173 Mazure, Fleischmann, et al., 1989; Steketee et al., 1996).

174

175 Table 1. The Yale-Brown Obsessive Compulsive Scale Self-Report (Y-BOCS-SR) with their 176 assigned item labels and mean (SD) scores per time point.

\begin{tabular}{|c|c|c|c|c|c|}
\hline \multirow[t]{2}{*}{ Item } & \multirow[t]{2}{*}{ Item label } & \multirow{2}{*}{$\begin{array}{l}\text { Mean (SD) } \\
\text { Baseline }\end{array}$} & \multirow{2}{*}{$\begin{array}{l}\text { Mean (SD) } \\
\text { Progress1 }\end{array}$} & \multirow{2}{*}{$\begin{array}{l}\text { Mean (SD) } \\
\text { Progress2 }\end{array}$} & \multirow{2}{*}{$\begin{array}{l}\text { Mean (SD) } \\
\text { Post }\end{array}$} \\
\hline & & & & & \\
\hline $\begin{array}{l}\text { Time occupied by } \\
\text { obsessive thoughts }\end{array}$ & O-Time & $2.66(1.15)$ & $2.34(1.04)$ & $2.16(1.05)$ & $1.76(0.98)$ \\
\hline $\begin{array}{l}\text { Interference due to } \\
\text { obsessive thoughts }\end{array}$ & O-Int & $2.39(1.06)$ & $1.99(0.93)$ & $1.77(0.95)$ & $1.41(0.89)$ \\
\hline $\begin{array}{l}\text { Distress associated } \\
\text { with obsessive } \\
\text { thoughts }\end{array}$ & O-Distr & $2.55(1.02)$ & $2.12(0.89)$ & $1.97(0.91)$ & $1.64(0.87)$ \\
\hline $\begin{array}{l}\text { Resistance against } \\
\text { obsessions }\end{array}$ & O-Res & $1.72(0.99)$ & $1.42(0.85)$ & $1.27(0.85)$ & $1.01(0.88)$ \\
\hline $\begin{array}{l}\text { Degree of control } \\
\text { over obsessive } \\
\text { thoughts }\end{array}$ & O-Cont & $2.32(0.96)$ & $1.91(0.83)$ & $1.74(0.81)$ & $1.42(0.79)$ \\
\hline $\begin{array}{l}\text { Time spent } \\
\text { performing } \\
\text { compulsive } \\
\text { behaviors }\end{array}$ & C-Time & $2.17(1.16)$ & $1.81(1.03)$ & $1.67(0.99)$ & $1.41(0.95)$ \\
\hline $\begin{array}{l}\text { Interference due to } \\
\text { compulsive } \\
\text { behaviors }\end{array}$ & C-Int & $2.11(1.16)$ & $1.74(1.04)$ & $1.54(0.97)$ & $1.28(0.95)$ \\
\hline $\begin{array}{l}\text { Distress associated } \\
\text { with compulsive } \\
\text { behavior }\end{array}$ & C-Distr & $2.36(1.06)$ & $2.05(0.93)$ & $1.91(0.93)$ & $1.56(0.90)$ \\
\hline $\begin{array}{l}\text { Resistance against } \\
\text { compulsions }\end{array}$ & C-Res & $1.89(1.06)$ & $1.40(0.84)$ & $1.23(0.85)$ & $0.95(0.82)$ \\
\hline
\end{tabular}


Introducing the Causal Graph Approach to Psychopathology

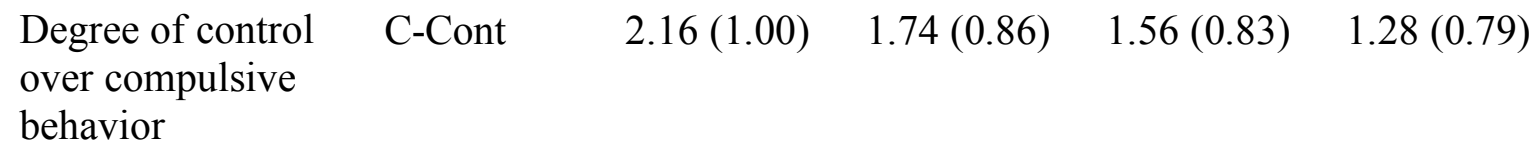

177

178

179

180

181

182

183

184

185

186

187

188

189

190

191

192

193

194

195

196

197

198

199

200

201

202

203

204

205

206

207

208

\section{Causal Graph Approach}

The proposed causal graph approach combines evidence for specific directed edges from three different sources: the ICP-algorithm (Meinshausen et al., 2016), the HICPalgorithm (Peters et al., 2017), and a literature study.

\section{Invariant Causal Prediction}

The ICP-algorithm (Meinshausen et al., 2016; Peters et al., 2016) is designed to handle the combination of observational and interventional data. The basic idea is that the conditional distribution of a single node (called the target node), given its direct causes, does not change if some manipulation takes place on variables other than the direct causes of a target node. Hence, for each node, the ICP-algorithm ascertains the subset of remaining nodes that cause the target node. The algorithm starts by selecting one node, which is the target node. The target node is then regressed on each possible subset of remaining nodes, ranging from an empty subset, to all remaining nodes in the graph. For each of these regressions, we obtain the residual distribution. This is then split according to an instrumental variable. This variable indicates which score (i.e., residual) belongs to which measurement time point. The algorithm then compares the residuals between every pair of measurements (4 in our illustration: before, twice during, and after treatment). If the residuals between a pair of measurements are deemed equal, we conclude that the causal relation between the subset of nodes and the target node is "invariant." After all subsets are examined, we draw directed edges from the intersection of all subsets to the target node. If, for a given subset of nodes, the residuals are similar across all measurements, then conditional on the direct causes of the target node, any manipulation will not change the distribution of the residuals of the target node when one conditions on the direct causes. This process is then repeated for all nodes in the graph. For a more detailed description of how the ICP-algorithm performs in a simulation study, see Kossakowski et al., (2019) and Meinshausen et al. (2016).

\section{Hidden Invariant Causal Prediction}


When we run the ICP-algorithm, we assume that we have measured all possible causes of every single node. However, it is very difficult to satisfy this assumption, especially

211 in psychopathology. Many aspects or events can affect features of OCD that are not captured

212 by the Y-BOCS-SR. For example, research has shown that the catastrophic misinterpretation

213 of obsessive thoughts (Rachman, 1997) can influence OCD symptoms. Also, OCD tends to

214 co-occur with other mental disorders such as anxiety or mood disorders (see Jones et al.,

215 2018; McNally et al., 2017; Ruscio et al., 2010; Ruzzano, Borsboom, \& Geurts, 2015).

216 Therefore, we ran the HICP-algorithm to account for the influence of hidden variables:

217 variables that are not measured, but that may affect those we did measure (Meinshausen et

218 al., 2016; Peters et al., 2017).

219 A hidden variable that affects both the cause and the target node inflates the relation

220 between the two variables if the hidden variable is not accounted for. When hidden variables

221 affect variables of interest, the ICP-algorithm can no longer estimate the causal relation

222 between two variables accurately. Accordingly, to estimate the true causal relation between

223 two variables, the HICP-algorithm employs an instrumental variable. An instrumental

224 variable influences only the cause, but not the target node. It is the same variable that we used

225 in the ICP-algorithm to split the data according to the different time points in the dataset. An

226 instrumental variable splits the data into four parts that correspond to the four assessments

227 that we have for our data. By comparing every pair of time points, the HICP-algorithm

228 partials out the effect of the hidden variable. For more information on the HICP-algorithm

229 and how it performs in a simulation study, see Kossakowski et al. (2019).

\section{Literature Search}

232 We aimed to complete the causal graph that results from the ICP and HICP-algorithm

233 by means of a literature study. Using Google Scholar and Web of Science, we searched for

234 articles on OCD. Within these studies, we looked for sentences that signify a causal relation

235 between specific aspects of OCD. For example, Marker, Calamari, Woodard, and Riemann

236 (2006, p.390) state that "Obsessive-compulsive disorder (OCD) is a common anxiety disorder

237 characterized by intrusive thoughts that are difficult to dismiss and that increase anxiety." A

238 sentence such as this expresses a causal relation from $O$-Time (time occupied by obsessive

239 thoughts) to the subsequent $O$-Distr (distress associated with obsessive thoughts). An

240 independent researcher unaware of our results double-checked our findings as a safeguard

241 against any possible subjectivity and confirmation bias. 
Results

\section{Literature Study}

The two researchers - the first two authors of this paper - found a total of 74 causal

246 relations in 15 articles and two books. Researcher R1, who also searched for the literature,

247 found 53 causal relations, and researcher R2 56. Of these causal relations, initially 21 were

248 found by both researchers in the same materials. The initial lack of overlap between the two

249 researchers was due to a different interpretation of the resistance items (O-Res and $C$-Res).

250 After deliberation between the two researchers, the overlap increased to 35 causal relations,

251 of which nine represent unique causal relations. We decided to include only those causal

252 relations found by both researchers. Supplementary Table S1 gives an overview of all the

253 edges that are in the causal graph with the corresponding source.

\section{Causal Graph}

Figure 2 presents the causal graph, in which both show the split results from the algorithms and the literature review (Figures $2 \mathrm{a}$ and $2 \mathrm{~b}$ ), and a causal graph (Figure 2c), where we combined the results from the ICP, the HICP-algorithm, and the literature review. Supplementary Table S1 gives an complete overview of the sources justifying inclusion of edges in Figure 2. The first characteristic that stands out in this causal graph is the lack of overlap between the different sources. Out of the 14 edges that were detected, three edges were detected by both the ICP-algorithm and the HICP-algorithm, and only two edges were detected by either an algorithm and the literature review. At the same time, many edges appeared at least two studies or sources; only five edges had only one source (algorithm or literature).

Another interesting aspect of this causal graph is that it depicts four cycles. The first

267 cycle (detected by both the ICP and the HICP algorithms) exists between $C$-Distr and $C$ -

268 Cont, which indicates that, when for example the score on C-Distr (distress associated with

269 compulsive behavior) changes, this causes the score on C-Cont (degree of control over

270 compulsive behavior) to change as well, and vice versa. This means that, on average, when

271 patients score higher on C-Cont, and thus have a lower level of control over their

272 compulsions, their score on C-Distr increases as well, and they on average experience more

273 distress associated with their compulsions.

274 The second cycle in Figure $2 \mathrm{a}$ and $2 \mathrm{c}$ is between $C$-Time and $C$-Int. This cycle, which

275 is only detected by the HICP-algorithm, may indicate that, when patients' score on C-Time

276 (time spent on compulsive behaviors) changes, this causes the score on C-Int (interference 
277 due to compulsive behaviors) to change as well, and vice versa. This means that, on average,

278 when patients score higher on C-Time, and thus spend more time performing compulsive

279 behaviors, their score on C-Int changes as well, and they on average experience more

280 interference in their daily life due to their compulsive behaviors.

281 The third cycle was between $O$-Distr and C-Time (Figure $2 \mathrm{~b}$ and c). This cycle

282 appeared only in the literature and suggests that when patients' score on $O$-Distr (distress

283 associated with obsessive thoughts) changes, this causes the score on C-Time (time spent on

284 compulsive behaviors) to change as well, and vice versa. This means that, on average, when

285 patients score higher on $O$-Distr, and thus on average experience more distress due to their

286 obsessive thoughts, their score on C-Time increases as well, and they spend more time

287 performing compulsive behaviors.

288 The fourth and last cycle was between $C$-Time and O-Time, and also only appeared in

289 the literature. This cycle indicate that, as patients' score on $O$-Time (time spent on obsessive

290 thoughts) changes, their score on C-Time (time spent on compulsive behaviors) changes as

291 well, and vice versa. Thus, spending more time on obsessive thoughts results in spending

292 more time on compulsive behaviors, which in turn leads to spending more time on obsessive

293 thoughts. 
$\odot$

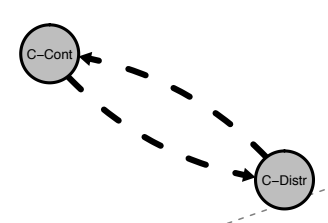

O-Cont

-

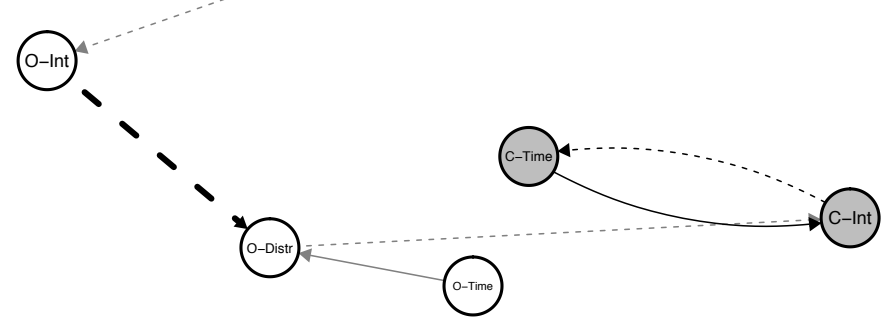

(a)

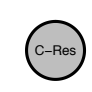

(c-con

(-)

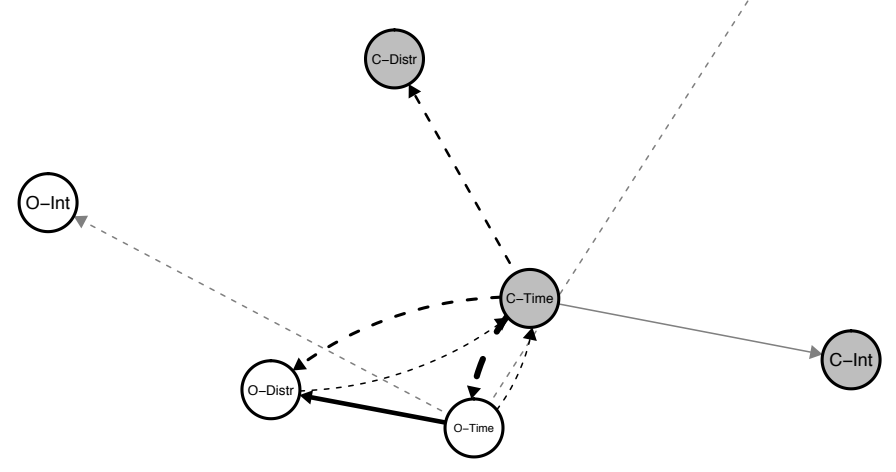

O-Res

(b)

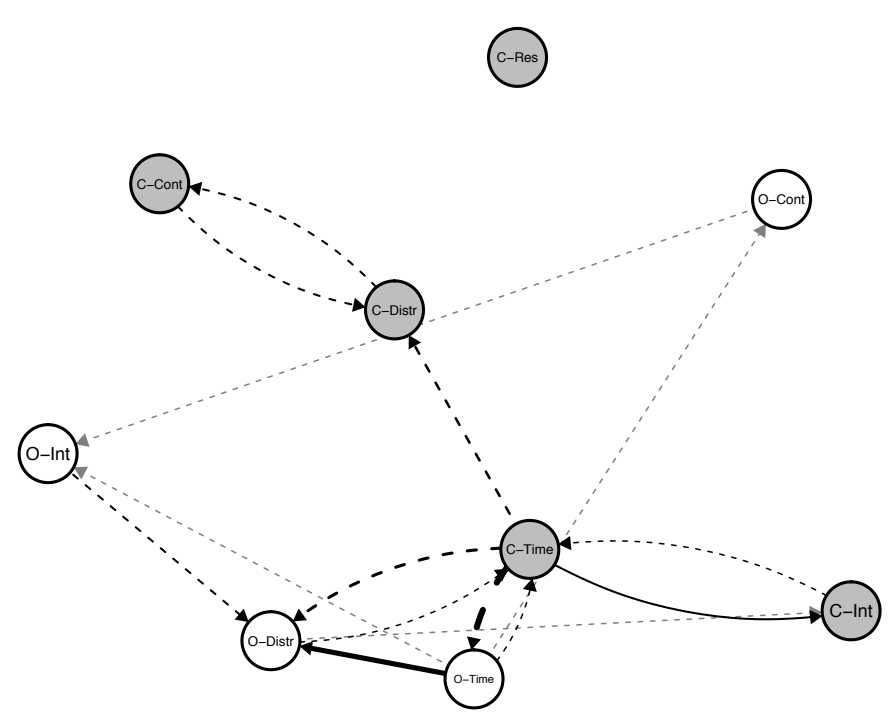

(-Res)

(c) 
296 Figure 2. Causal graph of the Y-BOCS-SR items, detected by the ICP and HICP algorithms

297 (a), found in the literature (b), and combined (c). Item labels are described in Table 1. White 298 nodes signify aspects of obsession, whereas gray nodes signify aspects of compulsions. Solid 299 edges are those detected by either of two algorithms and in the literature. Dashed edges are 300 those found with either of two algorithms, or in the literature. The edge thickness and color 301 saturation shows how often an edge is detected, the thicker and more saturated an edge, the 302 more often it was detected.

Interestingly, Figure 2 shows that the item $O$-Time is the cause of several other nodes and only received causal influence from the item C-Time. This could imply that the amount of time spent obsessing is the key driver of the causal system depicted in the graph, at least

307 for those individuals already diagnosed with OCD. This seems to confirm the (dominant) view that OCD starts with obsessive thoughts, although not everyone agrees with this (Gillan \& Sahakian, 2015). Lastly, two clinical features are disconnected from the rest of the causal graph, $O$-Res and $C$-Res. It is possible that this symptom is disconnected because it is part of the treatment (exposure and response prevention) that was given to the patients that were part 312 of this study.

Notably, only three causal relations connect the obsessive items with the compulsive ones, $O$-Time $\leftarrow \rightarrow C$-Time, $O$-Distr $\leftarrow \rightarrow C$-Time (both found in the literature) and the causal relation $O$-Distr $\rightarrow C$-Int (found with the ICP-algorithm), which in turn is also causally related to $C$-Time. This implies that there are only a few pathways whereby obsessive thoughts can lead to compulsions in patients, either directly or via some alternative path.

\section{Discussion}

In this exploratory study we introduced a causal graph approach and applied it to data of patients with OCD who completed the Y-BOCS-SR four times before, (twice) during, and

324 after treatment. Using two algorithms, we estimated a causal graph for OCD by combining observational and interventional data. The addition of interventional data enables the estimation of causal relations that are hard to detect with only observational data. The

327 resultant causal graph generated by the algorithms was complemented with a literature study.

328 Results exposed several cyclic patterns between features of OCD. These may signify

329 subsystems that maintain (parts of) the disorder throughout therapy. 
331 The ICP and HICP-algorithms did not estimate many causal relations between the items

332 of the Y-BOCS-SR. The ICP-algorithm estimated six causal relations, and the HICP-

333 algorithm estimated five causal relations, three of which were estimated by both algorithms.

334 In a graph where 45 different edges are possible, it is unclear whether the few causal

335 relations detected by these algorithms exhaust all of them or whether limited statistical power

336 may have rendered others difficult to detect. Indeed, Kossakowski et al. (2019) showed that

337 the ICP-algorithm can be quite conservative and will only use the intersection of all the

338 subsets that are investigated. This means that edges will only be estimated when a specific

339 edge appears in all the subsets that have significant causal relations with the target variable

340 (i.e., the item that is the recipient of the causal influence). The conservative character of the

341 ICP-algorithm is also shown by its high accuracy when the intervention is strong with a small

342 variance, which is an aspect of interventional data that is difficult to ascertain. At the same

343 time, Kossakowski and colleagues showed that the HICP-algorithm has a high sensitivity, but

344 only a moderate to high specificity depending on the number of edges in the graph and the

345 strength of the intervention. The lack of variance within the items could also have caused the

346 small number of causal relations to be estimated. However, as shown in Table 1, the standard

347 deviations of the items per time point are high enough to suggest otherwise.

348 The causal graph that shows the combined result of the ICP and HICP-algorithm

349 visualized a discrepancy between these two algorithms and the results of the literature study.

350 Only two causal relations that were detected by either of the two algorithms were confirmed

351 with the literature study. It is quite possible that the causal relations estimated with the

352 algorithms exist in the literature, but that we have not found the right studies that show

353 evidence for these relations. Even though many articles were found, not all articles explicitly

354 stated a causal relation between any two features of OCD, other than its two global, defining

355 symptoms: obsessions and compulsions. A search on Web of Knowledge uncovered more

356 than 5500 articles exist with the term "obsessive-compulsive disorder" in their title, so 17

357 different sources may not be representative of the existing literature on OCD. Another

358 disadvantage of the current literature study is that the used sources did not experimentally test

359 if one feature of OCD (e.g., time spent on obsessive thoughts) had a causal effect on another

360 (e.g., distress associated with compulsions).

361 The ICP and HICP-algorithm state that a causal relation between two variables can

362 only be invariant when the residual distributions between time points are equal. This means

363 that the causal relations found by the ICP and the HICP-algorithm reflect those causal 
364 relations that exist throughout therapy. Causal relations that change due to therapy are not 365 included, as these relations cannot be picked up by either the ICP-algorithm or the HICP366 algorithm. Although it is interesting to explore the causal relations that are not affected by 367 therapy, it can also be interesting to study those causal relations that vanish as a result of 368 therapy. Furthermore, in this study we examined patients who underwent treatment. We did 369 not have access to a control or waitlist condition for which we could run the same analyses 370 and compare the results. In future studies, adding a control condition and comparing results 371 could illuminate differences attributable to therapy. Another option would be to use therapy 372 as a dummy variable (when the data consists of an experimental and control condition) and 373 estimate the causal graph with that dummy variable, similar to the approach that Blanken, 374 van der Zweerde, van Straten, van Someren, and Borsboom (2019) used.

375 To our knowledge, this is the first study that estimates a causal graph for OCD using both 376 observational and interventional data. By combining results from different algorithms and 377 sources, we may uncover causal relations between features that otherwise are left unknown.

378 The result of this causal graph approach may potentially be used to set up intervention 379 strategies in a therapy setting. Even though we have to investigate to what extent this causal 380 graph approach is useful and meaningful for clinical practice, we do believe that the causal graph approach has a great potential for illuminating the causal structure of psychopathology.

\section{Author Contribution}

J.J.K, R.J.M., L.J.W., and H.L.J.M. jointly generated the idea for the study. J.J.K. programmed the study. B.C.R. was responsible for the data collection. J.J.K and L.J.B.O performed the literature study. J.J.K analyzed the data under the supervision of R.J.M, L.J.W and H.L.J.M. J.J.K. drafted the manuscript and L.J.B.O., R.J.M., B.C.R., L.J.W., and H.L.J.M provided critical revisions. All authors approved the final version of the paper for submission.

\section{Conflicts of Interest}

393 The authors declare that there were no conflicts of interest with respect to the authorship or 394 publication of this article.

\section{Funding}


Introducing the Causal Graph Approach to Psychopathology

397 J.J.K. is partly funded by the Research Priority Area Yield, part of the Research Institute of 398 Child development and Education, University of Amsterdam, the Netherlands, and by the 399 European Research Councel Consolidator Grant received by Prof. Denny Borsboom (grant 400 no. 647209).

401 
Blanken, T., van der Zweerde, T., van Straten, A., van Someren, E., \& Borsboom, D. (2019). Treatment Effects: A Demonstration in Co-Occurring Insomnia and Depression. Psychotherapy and Psychosomatics, 88, 52-54.

Borsboom, D. (2017). A network theory of mental disorders. World Psychiatry, 16, 5-13.

Borsboom, D., \& Cramer, A. O. J. (2013). Network Analysis: An Integrative Approach to the Structure of Psychopathology. Annual Review of Clinical Psychology, 9, 91-121.

Fontenelle, L. F., Mendlowicz, M. V, \& Versiani, M. (2006). The descriptive epidemiology of obsessive-compulsive disorder. Progress in Neuro-Psychopharmacology \& Biological Psychiatry, 30, 327-337.

Gillan, C. M., \& Sahakian, B. J. (2015). Which is the driver, the obsessions or the compulsions, in OCD? Neuropsychopharmacology, 40, 247-248.

Goodman, W. K., Price, L. K., Rasmussen, S. A., Mazure, C., Delgado, P., Heninger, G. R., \& Charney, D. S. (1989). The Yale-Brown Obsessive Compulsive Scale. II. Validity. Archives of General Psychiatry, 46, 1012-1016.

Goodman, W. K., Price, L. K., Rasmussen, S. A., Mazure, C., Fleischmann, R. L., Hill, C. L., ... Charney, D. S. (1989). The Yale-Brown Obsessive Compulsive Scale. I. Development, use, and reliability. Archives of General Psychiatry, 46, 1006-1011.

Holland, P. W. (1986). Statistics and Causal Inference. Journal of the American Statistical Association, 81, 945-960.

Jones, P. J., Mair, P., Riemann, B. C., Mugno, B. L., \& McNally, R. J. (2018). A network perspective on comorbid depression in adolescents with obsessive-compulsive disorder. Journal of Anxiety Disorders, 53, 1-8.

Kessler, R. C., Berglund, P., Demler, O., Jin, R., Merikangas, K. R., \& Walters, E. E. (2005). Lifetime Prevalence and Age-of-Onset Distributions of DSM-IV Disorders in the National Comorbidity Survey Replication. Archives of General Psychiatry, 62, 593-602.

Kossakowski, J. J., Waldorp, L. J., \& van der Maas, H. L. J. (2019). The Search for Causality: A Comparison of Different Techniques for Causal Inference Graphs.

Marker, C. D., Calamari, J. E., Woodard, J. L., \& Riemann, B. C. (2006). Cognitive selfconsciousness, implicit learning and obsessive-compulsive disorder. Anxiety Disorders, 20, 389-407.

Mataix-Cols, D., Fernandez de la Cruz, L., Nordsletten, A. E., Lenhard, F., Isomura, K., \& Blair Simpson, H. (2016). Towards an international expert consensus for defining 

treatment response, remission,
World Psychiatry, 15, 80-81.

McNally, R. J., Mair, P., Mugno, B. L., \& Riemann, B. C. (2017). Co-morbid obsessivecompulsive disorder and depression: a Bayesian network approach. Psychological Medicine, (7), 1204-1214.

Meinshausen, N., Hauser, A., Mooij, J. M., Peters, J., Versteeg, P., \& Buhlmann, P. (2016). Methods for causal inference from gene perturbation experiments and validation. Proceedings of the National Academy of Sciences, 113, 7361-7368.

Peters, J., Buhlmann, P., \& Meinshausen, N. (2016). Causal inference using invariant prediction: identification and confidence intervals.

Peters, J., Janzing, D., \& Schölkopf, B. (2017). Elements of Causal Inference: Foundations and Learning Algorithms. Cambridge, MA, USA: MIT Press.

Pinna, A., Soranzo, N., \& de la Fuente, A. (2010). From knockouts to networks: establising direct cause-effect relationships through graph analysis. PloS ONE, 5, e12912.

Rachman, S. (1997). A Cognitive Theory of Obsessions. Behavior Research and Therapy, $35,793-802$.

Ruscio, A. M., Stein, D. J., Chiu, W. T., \& Kessler, R. C. (2010). The epidemiology of obsessive-compulsive disorder in the National Comorbidity Survey Replication. Molecular Psychiatry, (15), 53-63.

Ruzzano, L., Borsboom, D., \& Geurts, H. M. (2015). Repetitive Behaviors in Autism and Obsessive-Compulsive Disorder: New Perspectives from a Network Analysis. Journal of Autism and Developmental Disorders, 45, 192-202.

Serra, R., Villani, M., \& Semeria, A. (2004). Genetic network models and statistical properties of gene expression data in knock-out experiments. Journal of Theoretical Biology, 227, 149-157.

Steketee, G., Frost, R., \& Bogart, K. (1996). The Yale-Brown Obsessive Compulsive scale: Interview versus self-report. Behavior Research and Therapy, 34, 675-684. 
465 Supplementary Table S1. Origin of all edges present in Figure 2.

\begin{tabular}{|c|c|c|}
\hline Edge & Algorithm & Literature \\
\hline O-Time - O-Int & & $\begin{array}{l}\text { Goodman, Rudorfer, \& Maser (2000, chapter 1, p. } \\
\text { 33) }\end{array}$ \\
\hline O-Time - O-Distr & ICP & $\begin{array}{l}\text { - Goodman, Rudorfer \& Maser (2000, chapter 1, p. } \\
\text { 33) } \\
\text { - Goodman, Rudorfer \& Maser (2000, chapter 7, p. } \\
\text { 239) } \\
\text { - Goodman, Rudorfer \& Maser (2000, chapter 11, } \\
\text { p. 395) } \\
\text { - Okasha (2002, chapter 1, p. 2) } \\
\text { - Jacoby, Leonard, Riemann, \& Abramowitz (2016, } \\
\text { p. 179) } \\
\text { - Janeck, Calamari, Riemann \& Heffelfinger (2003, } \\
\text { p. 182) } \\
\text { - Blakey, Abramowitz, Reuman, Leonard \& } \\
\text { Riemann (2017, p. 113) } \\
\text { - Marker, Calamari, Woodard \& Riemann (2006, p. } \\
\text { 390) }\end{array}$ \\
\hline O-Time - O-Cont & & $\begin{array}{l}\text { - Janeck, Calamari, Riemann \& Heffelfinger (2003, } \\
\text { p. 182) }\end{array}$ \\
\hline O-Time - C-Time & & $\begin{array}{l}\text { - Goodman, Rudorfer \& Maser (2000, chapter 6, p. } \\
223 \text { ) } \\
\text { - Goodman, Rudorfer \& Maser (2000, chapter 11, } \\
\text { p. 404) }\end{array}$ \\
\hline O-Int - O-Distr & $\mathrm{ICP}+\mathrm{HICP}$ & \\
\hline O-Distr - C-Time & & $\begin{array}{l}\text { - Najmi, Riemann, \& Wegner (2009, p. 494) } \\
\text { - Manos, Cahill, Wetterneck, Conelea, Ross \& } \\
\text { Riemann (2010, p. } 700)\end{array}$ \\
\hline O-Distr - C-Int & ICP & \\
\hline O-Cont - O-Int & $\mathrm{ICP}$ & \\
\hline C-Time - O-Time & & - Goodman, Rudorfer \& Maser (2000, chapter 6, p. \\
\hline
\end{tabular}


- Goodman, Rudorfer \& Maser (2000, chapter 6, p. 229)

- Goodman, Rudorfer \& Maser (2000, chapter 7, p. 241)

- Goodman, Rudorfer \& Maser (2000, chapter 11, p. 393)

- Goodman, Rudorfer \& Maser (2000, chapter 11, p. 399)

- Najmi, Riemann, \& Wegner (2009, p. 495)

- Hezel \& McNally (2016, p. 222)

- Hezel \& McNally (2016, p. 223)

- Hezel \& McNally (2016, p. 223)

- McNally \& Ricciardi (1996, p. 17)

- Tolin, Abramowitz, Przeworski, \& Foa (2002, p. 1256)

C-Time - O-Distr

- Goodman, Rudorfer \& Maser (2000, chapter 7, p. 241)

- Goodman, Rudorfer \& Maser (2000, chapter 11, p. 393)

- Goodman, Rudorfer \& Maser (2000, chapter 11, p. 395)

- Goodman, Rudorfer \& Maser (2000, chapter 11, p. 399)

- Najmi, Riemann \& Wegner (2009, p. 495)

C-Time - C-Int $\quad$ HICP Goodman, Rudorfer \& Maser (2000, chapter 1, p.

33)

C-Time - C-Distr

- Goodman, Rudorfer \& Maser (2000, chapter 1, p. 33)

- Goodman, Rudorfer \& Maser (2000, chapter 7, p. 239)

- Luchian, McNally, \& Hooley (2007, p. 1657)

- Okasha (2002, chapter 1, p. 3) 
Introducing the Causal Graph Approach to Psychopathology
C-Int - C-Time $\quad$ HICP
C-Distr $-\mathrm{C}$-Cont $\quad \mathrm{ICP}+\mathrm{HICP}$
C-Cont $-\mathrm{C}$-Distr $\quad \mathrm{ICP}+\mathrm{HICP}$

466

467

468 